Kansas State University Libraries

New Prairie Press

\title{
SHIGA TOXIN-PRODUCING ESCHERICHIA COLI IN MEAT: A PRELIMINARY SIMULATION STUDY ON DETECTION CAPABILITIES FOR THREE SAMPLING METHODS
}

\author{
Julie Couton \\ University of Nebraska-Lincoln, julie.couton@huskers.unl.edu \\ David Marx \\ University of Nebraska-Lincoln \\ John Luchaansky \\ USDA-ARS Wyndmoor, PA \\ Randall Phebus \\ Kansas State University \\ Anna Porto-Fett \\ USDA-ARS Wyndmoor, PA
}

See nextpage for additional authors

Part of the Agriculture Commons, Applied Statistics Commons, and the Food Processing Commons cc) (†) $\ominus$

This work is licensed under a Creative Commons Attribution-Noncommercial-No Derivative Works 4.0 License.

\section{Recommended Citation}

Couton, Julie; Marx, David; Luchaansky, John; Phebus, Randall; Porto-Fett, Anna; Sevart, Nicholas; Singh, Manpreet; and Thippareddi, Harshavardhan (2015). "SHIGA TOXIN-PRODUCING ESCHERICHIA COLI IN MEAT: A PRELIMINARY SIMULATION STUDY ON DETECTION CAPABILITIES FOR THREE SAMPLING METHODS," Conference on Applied Statistics in Agriculture. https://doi.org/10.4148/2475-7772.1090

This Event is brought to you for free and open access by the Conferences at New Prairie Press. It has been accepted for inclusion in Conference on Applied Statistics in Agriculture by an authorized administrator of New Prairie Press. For more information, please contact cads@k-state.edu. 


\section{Author Information}

Julie Couton, David Marx, John Luchaansky, Randall Phebus, Anna Porto-Fett, Nicholas Sevart, Manpreet Singh, and Harshavardhan Thippareddi 


\title{
SHIGA TOXIN-PRODUCING ESCHERICHIA COLI IN MEAT: A PRELIMINARY SIMULATION STUDY ON DETECTION CAPABILITIES FOR THREE SAMPLING METHODS
}

\author{
Julie Couton, Department of Statistics, University of Nebraska- Lincoln \\ David Marx, Department of Statistics, University of Nebraska- Lincoln \\ John Luchansky, Eastern Regional Research Center, USDA-ARS, Wyndmoor, PA \\ Randall Phebus, Food Science Institute, Kansas State University \\ Anna Porto-Fett, Eastern Regional Research Center, USDA-ARS, Wyndmoor, PA \\ Nicholas Sevart, Food Science Institute, Kansas State University \\ Manpreet Singh, Department of Food Sciences, Purdue University \\ Harshavardhan Thippareddi, Department of Poultry Science, University of Georgia
}

\section{ABSTRACT}

Contamination by Shiga Toxin-producing Escherichia coli (STEC) is a continuing concern for meat production facility management throughout the United States. Several methods have been used to detect STEC during meat processing, however the excessive experimental cost of determining the optimal method is rarely feasible. The objective of this preliminary simulation study is to determine which sampling method (Cozzini core sampler, core drill shaving, and N-60 surface excision) will better detect STEC at varying levels of contamination present in the meat. 1000 simulated experiments were studied using a binary model for rare occurrences to find the optimal method. We found that for meat contamination levels less than $0.1 \%$ or greater than $10 \%$ all sampling methods perform equally. At moderate levels of contamination (between $0.1 \%$ and $10 \%)$ core drill shaving and N-60 perform significantly better than Cozzini core sampler. However, there does not appear to be a significant difference between core drill shaving and $\mathrm{N}$ 60. This project was supported by an Agriculture and Food Research Initiative Competitive Grant no. 2012-68003-30155 from the USDA National Institute of Food and Agriculture.

Key Words: simulation study; binary response variable; rare occurrence model; STEC

\section{INTRODUCTION}

Contamination by Shiga Toxin-producing Escherichia coli (STEC) is a continued concern among meat production facilities throughout the United States. A variety of methods have been developed to detect STEC during meat processing, however full experimental implementation is costly.

This simulation study focuses on the detection capabilities of three of these methods: Cozzini core sampler, core drill shaving, and N-60 surface excision. While all three methods perform well when high percentages of contaminated meat are present, results differs across methods as the percentage of contaminated meat decreases. The primary research question is which of the three methods is best able to detect STEC with varying levels of contamination present among the meat. 


\section{MEAT PROCESSING AND SAMPLING}

Typically in production, the carcass is processed and cuts of meat are sent down a conveyor belt then collected in large bins, called combos. Combos are selected at random to be tested. One of the sampling methods is used to collect cuts of meat which are then ground together and enriched before undergoing contamination analysis. If STEC is found to be present in the sample, all of the meat in the combo cannot be processed for human consumption and the bin is considered to be contaminated (1) otherwise it is considered to not be contaminated (0). Contamination is typically found on the surface of the individual cuts of meat.

\section{Loading Process- Simulation}

In this simulation study, all meat was considered to be the same size $\left(2000 \mathrm{~cm}^{3}\right)$ with varying orientation determined at random. See Figure 1 in the Appendix.

Meat was loaded into a combo ( $80 \mathrm{~cm}$ long $\times 80 \mathrm{~cm}$ wide $\times 100 \mathrm{~cm}$ tall) layered from the bottom until the combo was filled. The randomly assigned orientations acted as a packing mechanism to ensure that all pieces of generated meat would fit within the combo. Once filled the combos held on average 225-250 cuts of meat. Figure 2 in the Appendix shows a transverse view of the combo from above once it had been filled.

The combo was then chosen to have one of six levels of contamination: $0.1 \%, 1 \%, 3 \%, 5 \%, 10 \%$, or $15 \%$. Using the assigned level of contamination, the cuts of meat within each combo were then uniformly contaminated at random. Whether the combo contains 1 cut or 100 cuts of meat that are contaminated the entire combo is deemed to be unfit for human consumption.

This process was repeated to form 1000 combos for each level of contamination resulting in 6000 total combos used for analysis. The three sampling methods discussed in a subsequent section were used on each combo.

\section{Assumptions}

Several assumptions were made in the process of this preliminary simulation study. First, the meat is assumed to be contaminated by STEC at random; no clustering mechanism was established for cuts of meat from the same carcass. Additionally all cuts of meat were assumed to be the same size. As mentioned above, after random contamination the cuts were assumed to be uniformly contaminated. If a piece of contaminated meat was sampled, it was assumed that the contamination was captured and then perfectly detected.

\section{Sampling Methods}

1. N-60 Surface Excision

With N-60 Surface Excision (N60), 60 samples are drawn from the surface of the combo (see Figure 3 ). This method is commonly used in practice, because it is the simplest to implement. A variant of this method is $\mathrm{N}-90$ where an additional 30 samples are drawn from the surface. 
In Figure 2 in the appendix, the transect shows 40 cuts of meat. To obtain N60, 60 cuts of meat on the top of the combo were sampled, meaning that sampling continued into the second layer of meat. This is commonly done in practice.

\section{Cozzini Core Sampler}

The Cozzini core sampler (Cozz) is $4.4 \mathrm{~cm}$ in diameter and permeates to the bottom of the combo. This core samples both the surface and interior of the individual cuts of meat (see Figure 4). In this method, five cores are drawn from the combo: one from each corner and one from the center. Within each of these areas, the specific placement of the sampler is chosen at random (see Figure 5). These cores are then combined and collectively act as a sample from the combo.

\section{Core Drill Shaving}

The core drill shaving method (Core) utilizes a shaver that is $3.2 \mathrm{~cm}$ in diameter. Although the shaver is shorter in length than the Cozzini core, reaching only halfway down into the combo, it collects samples from primarily the surface area of each cut (see Figure 6). While the Cozzini permeates through the cuts of meat, the Core shaves all meats surrounding it, at times traveling horizontally rather than strictly vertically. Like the Cozzini, 5 shavings are drawn from the combo, one from each corner with an additional shaving collected in the center (see Figure 7). These shavings are combined and considered to be a single sample from the combo.

\section{Basic Data Summary}

Due to our assumptions, it is apparent that a large component of successful detection of STEC is the number of pieces of meat sampled.

\begin{tabular}{|lrr|}
\hline \multicolumn{3}{|c|}{ Number of Pieces Sampled } \\
\hline Method & Mean & \multicolumn{1}{c|}{ Std Dev } \\
\hline Cozz & 37.30 & 2.46 \\
\hline N60 & 60.00 & 0.00 \\
\hline Core & 63.78 & 2.30 \\
\hline
\end{tabular}

Table 1: Average number of pieces sampled within each combo.

We assumed that meat is contaminated strictly at random (without a clustering mechanism), thus the more pieces of meat that are sampled the better the detection will be. In Table 1, Cozz is sampling fewer pieces of meat on average than the other methods, while N60 and Core are performing similarly on average.

\section{ANALYSIS AND RESULTS}

Recall that the sampling method either detects contamination (1) or does not (0). Following the notation of Stroup (2013), this binary response variable was modeled as follows: 


$$
\begin{gathered}
y_{i j k} \mid b(c)_{j k} \sim \operatorname{Binary}\left(\pi_{i j k}\right), \quad \eta_{i j k}=\operatorname{logit}\left(\pi_{i j k}\right)=\log \left(\frac{\pi_{i j k}}{1-\pi_{i j k}}\right) \\
\eta_{i j k}=\eta+m_{i}+c_{j}+m c_{i j}+b(c)_{j k}, \quad b(c)_{j k} \sim \operatorname{Gaussian}\left(0, \sigma_{B}^{2}\right)
\end{gathered}
$$

Where $\eta_{i j k}$ is the linear predictor, $m_{i}$ represents the main effect of sampling method $i, c_{j}$ represents the main effect of contamination level $j(0.1 \%, 1 \%, 3 \%, 5 \%, 10 \%$, or $15 \%)$, and $m c_{i j}$ represents their interaction. Observed response $y_{i j k}$ represents the contamination ( 0 or 1 ) which is conditioned on the random effect $b(c)_{j k}$ because the observation occurs within each combo or block, $b_{k}$, assigned a level of contamination. Here, combos are considered to be random due to the nature of the testing procedure in reality (each combo is a sample from a large population of combos) which was emulated in the simulation process. Combos are assumed to be normally distributed with mean 0 and variance $\sigma_{B}^{2}$. All simulated replications were combined and analyzed using SAS PROC GLIMMIX'⿳一.⿲丿丨丶⿴囗十 .

The analysis results can be found in the appendix in Table 2, and are plotted in Figure 8 below. Of note is that the interaction between $m$ and $c$ is statistically significant ( $p$-value $<0.0001$ ).

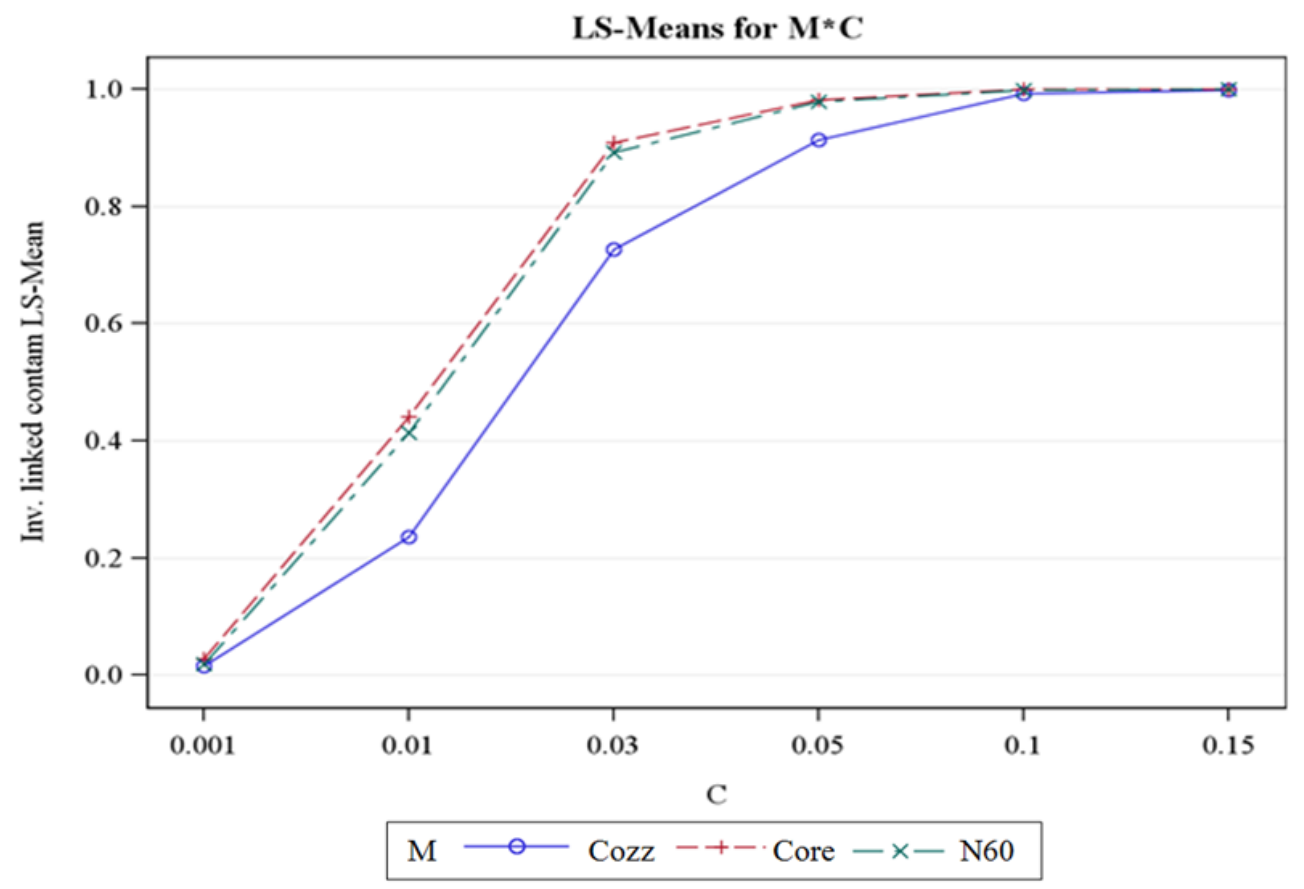

Figure 8: A plot of the mean estimates for each method considered across the different levels of contamination for the full model (e.g. 0.001 represents the case where $0.1 \%$ of meat is contaminated, etc.).

The plot makes it apparent that at the lowest level of contamination (0.001) the methods are equally ineffective at detecting contamination. At high levels of contamination ( 0.1 and 0.15 ) the methods are equally successful at detecting contamination. Thus, the most noticeable differences occur across moderate levels of contamination $(0.01,0.03$, and 0.05$)$. 
Restricting the analysis to moderate levels of contamination, we again find that the interaction between $m$ and $c$ is statistically significant ( $p$-value $=0.0042$, Table 3 in the Appendix). These results are considered to be statistically significant, which largely occurred due to the large number of replications.

Looking at the simple effects (Table 4 in the Appendix), we can see that across all considered levels of contamination in this model, Cozz performs significantly worse than the other two methods. $\mathrm{N} 60$ and Core do not perform significantly different from each other.

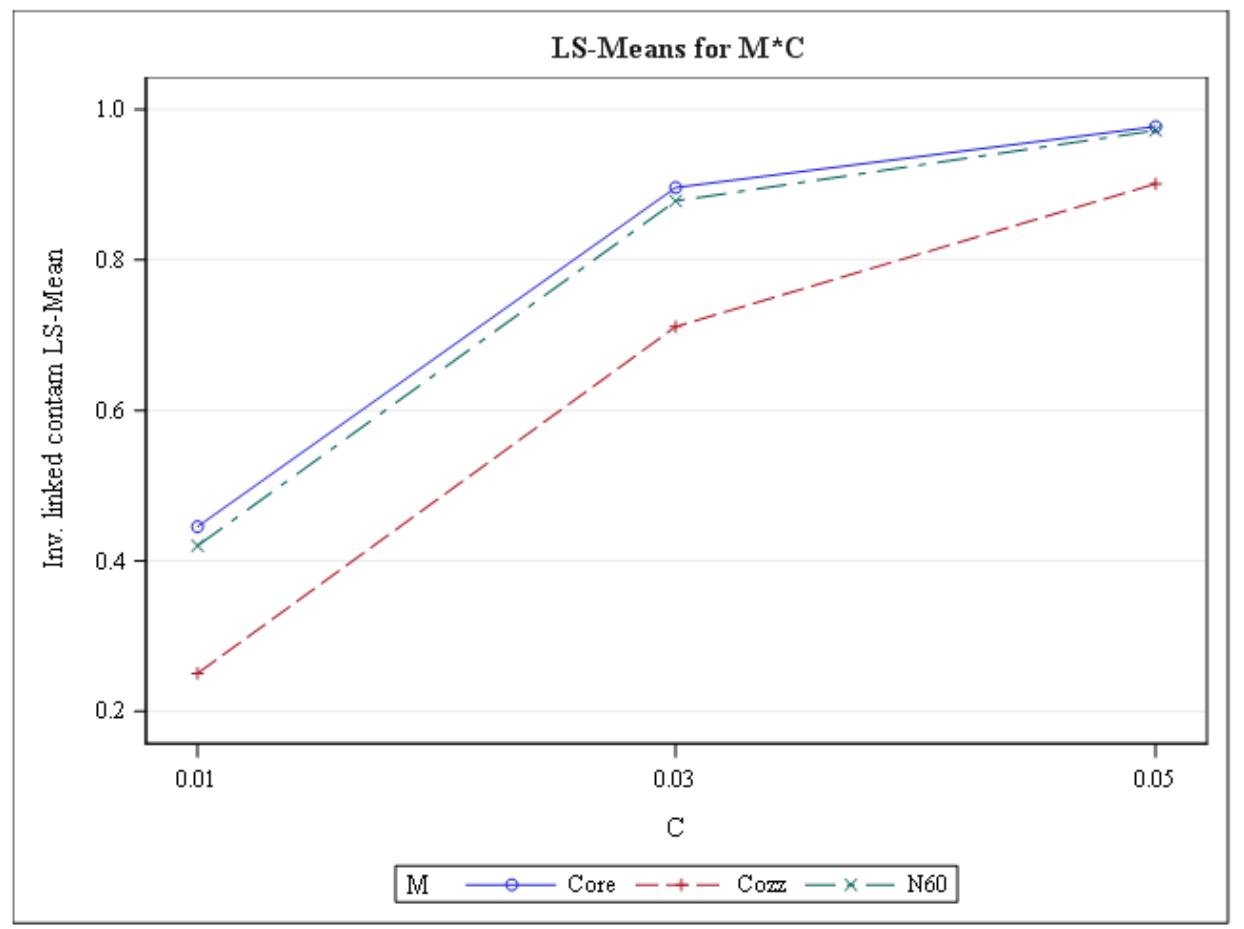

Figure 9: A plot of the mean estimates for each method considered across moderate levels of $C$ for the full model. Because the levels for $c$ under consideration have been reduced, this will be referred to as the reduced model (e.g. 0.01 represents the case where $1 \%$ of meat is contaminated, etc.).

The methods of analysis used thus far are sufficient to answer the initial research question. However, further analysis is warranted in regards to estimation. Because this data was simulated, we can determine how well the methods estimated the true proportion of contaminated combos. The estimation capabilities are explored in Figure 10 below. 


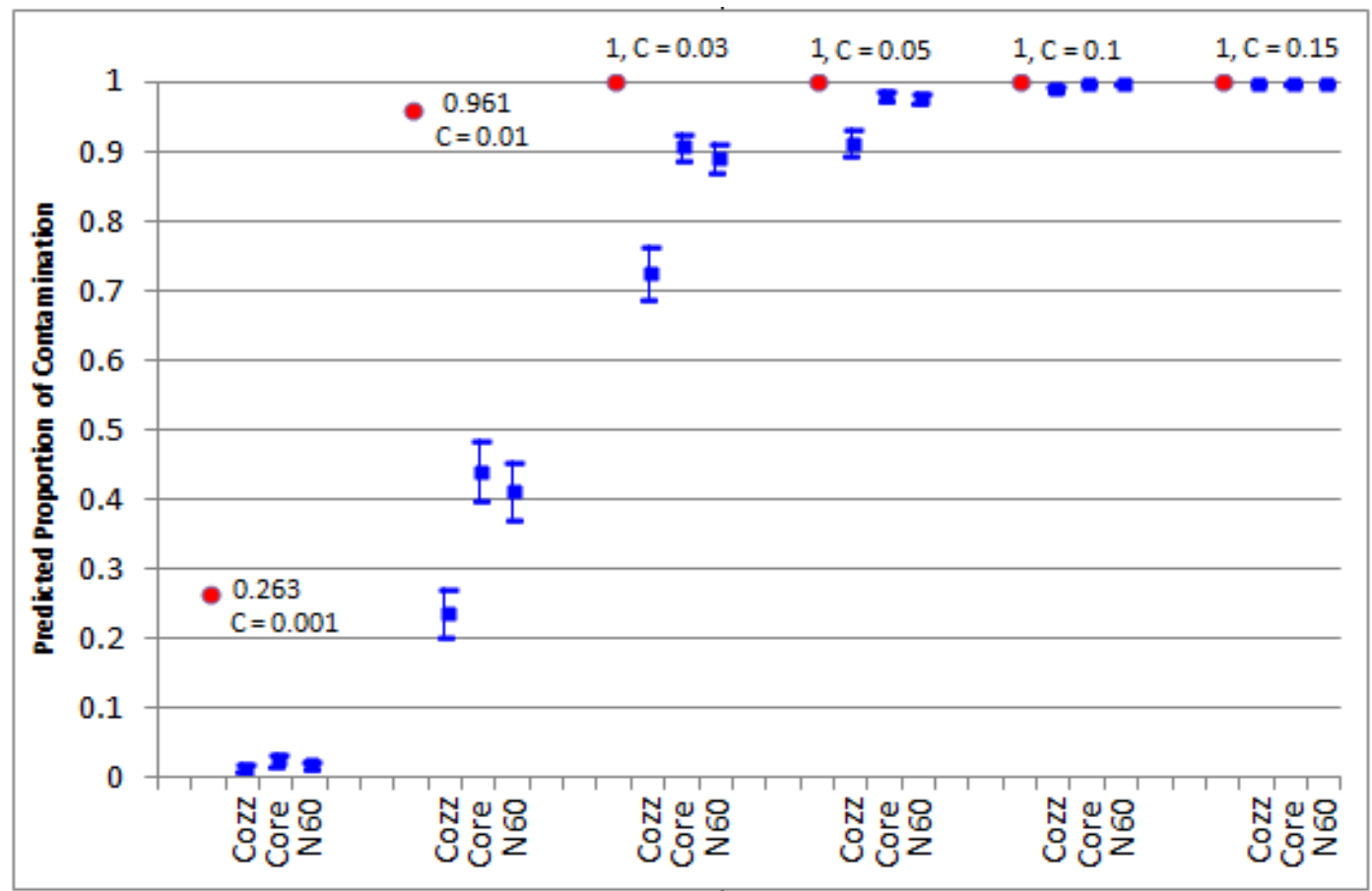

Figure 10: This plot, shows the confidence interval bounds for each method (Cozz, Core, N60) at each level of contamination, $C(C=0.001=0.1 \%$ of the meat is contaminated, etc.). The value next to the dot is the true proportion of combos that contained at least one piece of contaminated meat.

These are $95 \%$ confidence intervals, using a normal approximation and the standard errors given in Table 5 in the Appendix. It is apparent that as the true percentage of contaminated meat decreases, the sampling methods ability to detect the contamination drops dramatically.

\section{DISCUSSION}

- Answering the research question.

This research shows that if the contamination is less than $0.01 \%$ all sampling methods will struggle to detect contamination and at levels greater than $10 \%$ all methods are effectively detecting the contamination. Over moderate levels of contamination (between $1 \%$ and $5 \%$ ) core drill shaving and $\mathrm{N}-60$ surface excision perform significantly better than Cozzini core sampler at all levels. There does not appear to be a significant difference between core drill shaving and $\mathrm{N}-60$.

Revisiting the descriptive statistics from Table 1, these results are as expected. The sampling methods that collect samples from more pieces of meat will have a higher chance of detecting the contamination. Both Core and $\mathrm{N} 60$ were sampling significantly more pieces of meat per combo than Cozz which explains the results presented above. So we can conclude that sampling more meat will lead to higher detection capabilities. 
However, this conclusion is valid for this simulation only. The result would likely change if the assumptions made in the simulation process were altered, to incorporate more realistic conditions. Specifically, the simulation warrants the incorporation of different sizes of meat and the inclusion of a clustering mechanism. In production facilities, each piece of meat is not contaminated completely at random. If there is contamination on the hide of the carcass, the contamination is more likely to occur on meat from that carcass. Inclusion of these components is paramount before any firm conclusions can be drawn in regards to these sampling methods.

- Looking beyond the research question towards estimation capabilities.

The results of this study show that as the presence of contamination $(c<0.05)$ decreases, the sampling methods ability to capture this contamination drops dramatically. It is unclear if this trend will be present as the simulation assumptions are relaxed.

By incorporating the estimation into this preliminary simulation study, we have come to realize that simply determining which method is superior is far from adequate. We must ensure that the sampling methods result in accurate determination of contamination due to the high consequences of misclassifying a combo as safe for consumption.

\section{FUTURE RESEARCH}

This study is intended as a first step in a sequence of simulation studies that further incorporate realistic scenarios with less restrictive assumptions. Specifically, incorporating various sized meats (proportional to those that are currently processed in the production facilities) and a clustering mechanism. Finally, assuming perfect detection (no false positives or false negatives) is not realistic. This should also be incorporated into subsequent simulations and analyses.

\section{ACKNOWLEDGEMENTS}

This project was supported by an Agriculture and Food Research Initiative Competitive Grant no. 2012-68003-30155 from the USDA National Institute of Food and Agriculture.

\section{REFERENCES}

Stroup, W.W. 2013. Generalized Linear Mixed Models. Boca Raton, FL. CRC Press. 


\section{APPENDIX}

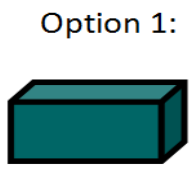

$20 \times 10 \times 10$
Option 2:

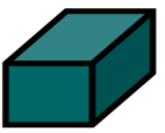

$10 \times 20 \times 10$
Option 3:

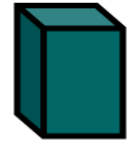

$10 \times 10 \times 20$

Figure 1: Each of these options represents the possible orientation assigned at random to each cut of meat.

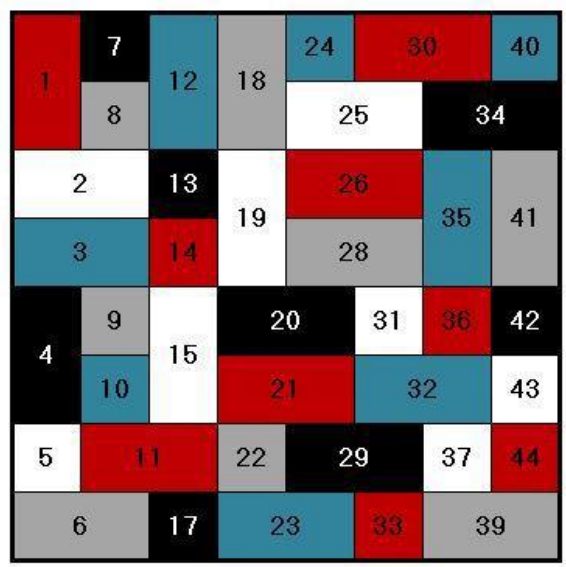

Figure 2: A transverse view of a section of the combo from above. Each rectangle or square represents a different piece of meat.

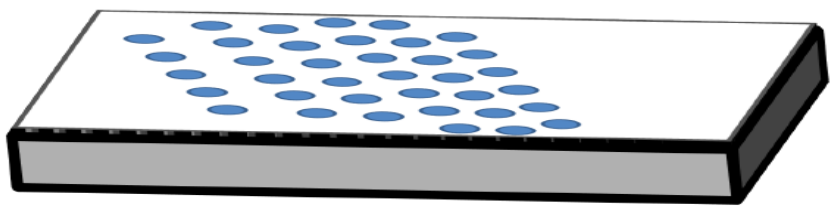

Figure 3: A simplified depiction of the surface of the combo and the $\mathrm{N} 60$ sampling method is shown here.

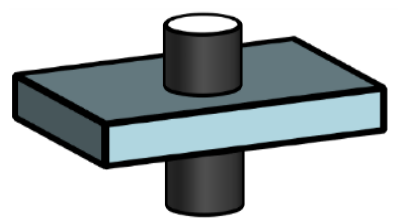

Figure 4: Here, the box is used to depict an individual cut of meat, where the Cozzini core sampler (the cylinder) is permeating through the cut. 


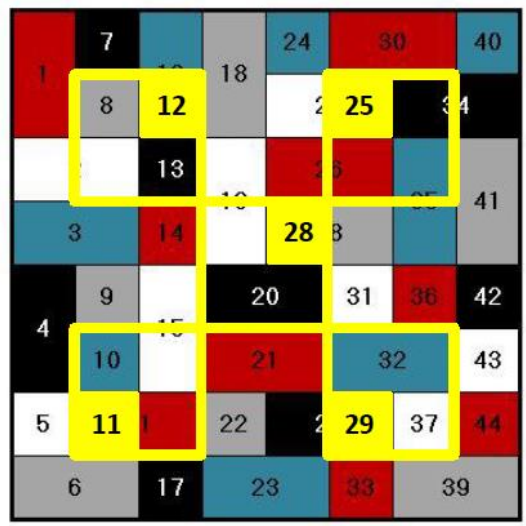

Figure 5: Here, the large yellow squares represent the areas of interest (the corners and the center). Within each location the placement of the Cozzini core sampler was chosen at random. Here the sampler would be placed above cut 11 and permeate through to the bottom of the combo. Similarly for cuts $12,25,28$ and 29.

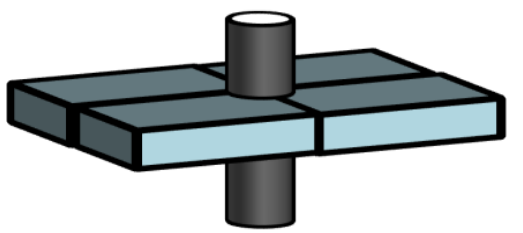

Figure 6: The core drill shaver (seen as the black cylinder) shaves all of the cuts of meat (seen as the boxes) surrounding it, obtaining a core that is primarily made up of surface area meat.

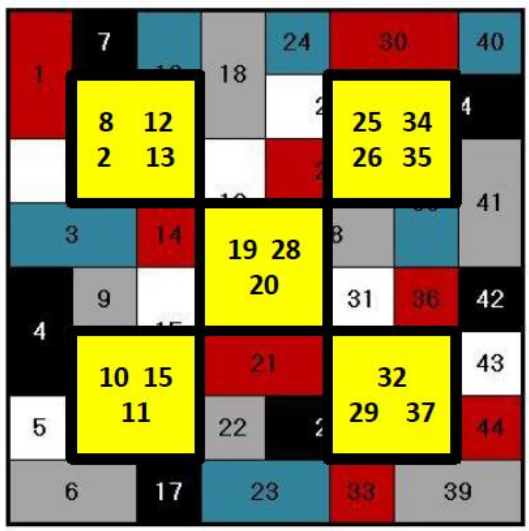

Figure 7: The core drill shaver method considers the large yellow squares as areas of interest, similar to the Cozzini. However, because the shaver captures surrounding meat placing the shaver at the center of the yellow squares leads to the collection of all surrounding pieces of meat. Thus in the top left area, cuts 2, 8, 12 and 13 are all collected. Bear in mind that this shaver only reaches halfway into the combo. 


\begin{tabular}{|lrrrr|}
\hline \multicolumn{5}{c|}{ Type III Tests of Fixed Effects } \\
\hline Effect & Num DF & Den DF & F Value & Pr > F \\
\hline C & 5 & 5994 & 332.41 & $<.0001$ \\
M & 2 & 11988 & 9.21 & 0.0001 \\
\hline M*C & 9 & 11988 & 13.14 & $<.0001$
\end{tabular}

Table 2: This test is for the full model analysis, showing that the interaction between contamination level and sampling method is statistically significant.

\begin{tabular}{|lrrrr|}
\hline \multicolumn{5}{c|}{ Type III Tests of Fixed Effects (Reduced) } \\
\hline Effect & Num DF & Den DF & F Value & Pr > F \\
C & 2 & 2997 & 491.48 & $<.0001$ \\
M & 2 & 5994 & 139.7 & $<.0001$ \\
\hline M*C & 4 & 5994 & 3.81 & 0.0042
\end{tabular}

Table 3: This is for the model considered under moderate levels of contamination. This is apparent by the reduced degrees of freedom. Due to the large number of replications, we again see a significant interaction.

\begin{tabular}{|c|c|c|c|c|c|c|c|}
\hline \multicolumn{8}{|c|}{ Simple Effect Comparisons of $\mathbf{M}^{\star} \mathbf{C}$ Least Squares Means By $\mathbf{C}$} \\
\hline \multirow{2}{*}{$\begin{array}{l}\text { Simple Effect } \\
\text { Level }\end{array}$} & \multicolumn{7}{|c|}{ Standard } \\
\hline & M & $\mathbf{M}$ & Estimate & Error & DF & t Value & $\operatorname{Pr}>|t|$ \\
\hline C 0.01 & Core & Cozz & 0.8769 & 0.1082 & 5994 & 8.11 & $<.0001$ \\
\hline C 0.01 & Core & N60 & 0.1067 & 0.1033 & 5994 & 1.03 & 0.3018 \\
\hline C 0.01 & Cozz & N60 & -0.7702 & 0.108 & 5994 & -7.13 & $<.0001$ \\
\hline C 0.03 & Core & Cozz & 1.2501 & 0.1235 & 5994 & 10.12 & $<.0001$ \\
\hline C 0.03 & Core & N60 & 0.1805 & 0.1313 & 5994 & 1.37 & 0.1692 \\
\hline C 0.03 & Cozz & N60 & -1.0695 & 0.1198 & 5994 & -8.93 & $<.0001$ \\
\hline C 0.05 & Core & Cozz & 1.5436 & 0.1895 & 5994 & 8.14 & $<.0001$ \\
\hline C 0.05 & Core & N60 & 0.1502 & 0.2241 & 5994 & 0.67 & 0.5026 \\
\hline C 0.05 & Cozz & N60 & -1.3933 & 0.181 & 5994 & -7.7 & $<.0001$ \\
\hline
\end{tabular}

Table 4: The following shows the simple effects. Most notable is that Core and N60 always perform significantly better than Cozz, while across each contamination level, Core and N60 never perform significantly different from each other. Estimated differences and standard errors are presented on the model scale. 


\begin{tabular}{|c|c|c|c|c|c|c|}
\hline $\mathbf{M}$ & C & DF & t Value & $\operatorname{Pr}>|t|$ & Mean* & $\begin{array}{c}\text { SE } \\
\text { Mean* }\end{array}$ \\
\hline Cozz & 0.001 & 11988 & -20.64 & $<.0001$ & 0.01486 & 0.00298 \\
\hline Cozz & 0.01 & 11988 & -12.28 & $<.0001$ & 0.2365 & 0.01723 \\
\hline Cozz & 0.03 & 11988 & 10.42 & $<.0001$ & 0.7259 & 0.01860 \\
\hline Cozz & 0.05 & 11988 & 19.74 & $<.0001$ & 0.9135 & 0.00943 \\
\hline Cozz & 0.1 & 11988 & 18 & $<.0001$ & 0.9927 & 0.00199 \\
\hline Cozz & 0.15 & 11988 & 9.7 & $<.0001$ & 0.999 & 0.00072 \\
\hline Core & 0.001 & 11988 & -21.69 & $<.0001$ & 0.02716 & 0.00436 \\
\hline Core & 0.01 & 11988 & -2.67 & 0.0075 & 0.4414 & 0.02170 \\
\hline Core & 0.03 & 11988 & 19.61 & $<.0001$ & 0.9084 & 0.00973 \\
\hline Core & 0.05 & 11988 & 21.19 & $<.0001$ & 0.9812 & 0.00344 \\
\hline Core & 0.1 & 11988 & 7.61 & $<.0001$ & 0.9995 & 0.00048 \\
\hline Core & 0.15 & 11988 & 0.1 & 0.9173 & 1 & 0.00000 \\
\hline N60 & 0.001 & 11988 & -21.16 & $<.0001$ & 0.01882 & 0.00345 \\
\hline N60 & 0.01 & 11988 & -3.95 & $<.0001$ & 0.4135 & 0.02143 \\
\hline N60 & 0.03 & 11988 & 18.74 & $<.0001$ & 0.8914 & 0.01087 \\
\hline N60 & 0.05 & 11988 & 21.43 & $<.0001$ & 0.9781 & 0.00379 \\
\hline N60 & 0.1 & 11988 & 9.75 & $<.0001$ & 0.999 & 0.00069 \\
\hline $\mathrm{N} 60$ & 0.15 & 11988 & 0.07 & 0.9442 & 1 & 0.00000 \\
\hline
\end{tabular}

Table 5: This table shows the mean and standard error (SE) estimates based on the full model for each combination of Method $(M)$ and level of Contamination $(C)$. These values were used to approximate $95 \%$ confidence intervals, by multiplying each SE by two. 